\title{
THROMBOCYTOPENIA IN RELATION WITH PLASMODIUM VIVAX
}

\section{MALARIA}

K. Kuladeepa Ananda Vaidya, Pradeep Vernekar

1. Assistant Professor, Department of Pathology, SIMS \& RC, Surathkal, Karnataka.

2. Assistant Professor, Department of General Medicine, SIMS \& RC, Surathkal, Karnataka.

\section{CORRESPONDING AUTHOR}

Dr. K. Kuladeepa Ananda Vaidya, Assistant professor, Dept of Pathology, SIMS\&RC, Srinivas Nagar, Mukka, Surathkal, Karnataka - 575021.

E-mail: vaidyakuldeep@gmail.com,

\begin{abstract}
Malaria is a major health problem in the tropics with increased morbidity and mortality. Plasmodium vivax is the most frequent and widely distributed cause of recurring malaria worldwide. Plasmodium vivax malaria is endemic infection in India and is commonly associated with haematological abnormalities, Thrombocytopenia is frequently observed in vivax malaria but the exact mechanism has not been elucidated. It has been increasingly observed that P. vivax malaria, which was otherwise considered to be benign malaria, with a low case-fatality ratio, can also occasionally result in severe disease as with P. falciparum malaria. This study was undertaken to correlate the presence and severity of thrombocytopenia with P. vivax malaria.
\end{abstract}

KEY WORDS: Plasmodium vivax, Malaria, Thrombocytopenia.

INTRODUCTION: Each year there are more than 250 million cases of malaria, killing between 1 to 3 million people [1]. Countries in which malaria had been previously eradicated or adequately controlled have recently seen a resurgence of malaria [2]. Hematological abnormalities have been observed in patients with malaria, anemia and thrombocytopenia being the most common [3]. Malaria is a common infection in most parts of India and is commonly associated with mild thrombocytopenia [4]. Profound thrombocytopenia is a wellrecognized complication of falciparum malaria but has been less well described in vivax malaria.[5] Thrombocytopenia seen in complicated falciparum malaria is due to disseminated intravascular coagulation along with platelet endothelial activation, but the one seen in uncomplicated malaria like P. vivax has multifactorial etiology. Few postulated mechanisms are macrophage activation leading to platelet destruction, increased levels of cytokines, immunological destruction due to antiplatelet IgG, oxidative stress, shortened platelet life span in peripheral blood and sequestration in nonsplenic areas and partly due to psuedothrombocytopenia due to clumping of platelets [6]

MATERIALS AND METHODS: This is a prospective study conducted at a tertiary referral hospital at coastal Karnataka. Study was conducted over a period of 6 months. A total of 150 patients who were hospitalized after confirmation of diagnosis of malaria by examination of peripheral smear stained with leishmen stain [Fig1, 2] or by detecting parasite by fluorescent technique (MPFT). Smear has been considered negative if no parasite detected on examination under 100 high power fields. Platelet counts have been performed by automated haematology analyser. Patients with decreased platelet count (thrombocytopenia) were re-evaluated by 
manual method. Thrombocytopenia was defined as platelet count of less than 150,000 cells/ $\mathrm{cmm}$. Patients were divided into three subgroups based on platelet count. Thrombocytopenia was considered severe if $<50,000$ cells $/ \mathrm{cmm}$, moderate if 50,000-100,000 cells/cmm, and mild if $100,000150,000$ cells $/ \mathrm{cmm}$.

RESULTS: We studied 150 cases of acute vivax malaria; The commonest presenting manifestations were fever with chills and rigors. Out of 150 cases 133 cases had thrombocytopenia, which was the most common haematological finding. None had bleeding manifestations from any site. Anaemia was present in 20 cases and splenomegaly was seen in two of the cases. Thrombocytopenia was seen as an early laboratory finding in 133 cases at the time of admission with fever of not more than 5 days. Platelet counts reverted to normal on treatment. Other causes of thrombocytopenia were ruled out by complete history and physical examination and dengue serology wherever required.

Mild thrombocytopenia $(1,00,000-150,000 / \mathrm{cmm})$ was seen in $42.8 \%$ of patients positive for P. Vivax with thrombocytopenia, $6.8 \%$ patients had severe thrombocytopenia with platelet counts less than $50,000 / \mathrm{cmm}$. (Table 3 ). The average duration of stay in the hospital for all patients was $5 \pm 2$ days.

Table1: Age and Sex distribution for P. Vivax

\begin{tabular}{|c|c|c|c|}
\hline Age (in years) & Male & Female & Total \\
\hline$<10$ years & - & 1 & 1 \\
\hline $10-20$ & 45 & 7 & 52 \\
\hline $21-30$ & 58 & - & 58 \\
\hline $31-40$ & 21 & 2 & 14 \\
\hline $41-50$ & 12 & - & 2 \\
\hline $51-60$ & 2 & - & 1 \\
\hline $61-70$ & 1 & 1 & 1 \\
\hline Above 70 & - & & \\
\hline
\end{tabular}


Table 2: P. Vivax with Thrombocytopenia

\begin{tabular}{|l|l|}
\hline Thrombocytopenia & Number of patients \\
\hline Present & $133(88.67 \%)$ \\
\hline Absent & $17(11.33 \%)$ \\
\hline Total & 150 \\
\hline
\end{tabular}

Table3: Severity of thrombocytopenia

\begin{tabular}{|c|c|}
\hline Severity & Number of cases \\
\hline Mild & $57(42.8 \%)$ \\
\hline Moderate & $67(50.4 \%)$ \\
\hline Severe & $9(6.8 \%)$ \\
\hline
\end{tabular}

DISCUSSION: Malaria infects mankind globally. According to World Health Organization assessment, about $40 \%$ of the world population is at risk of developing malaria. About 300-500 million people are infected with it. Every year about 2 million people die due to malaria and its complications. The highest mortality is in Africa, mainly in young children.[7]. Plasmodium vivax is the most widely distributed human malaria parasite with an at risk population of 2.5 billion persons. Although the exact burden of disease caused by P. vivax infection is still a matter of debate, this parasite causes approximately 100-300 million clinical cases each year.[8]. The trend of disease with plasmodium vivax malaria is changing. It is increasingly recognized that serious and life threatening complications can occur with vivax malaria. [9]

The mechanism of thrombocytopenia in malaria is not clearly known. Fajardo and Tallent in 1974 demonstrated P. vivax within platelets by electron microscopy and suggested a direct lytic effect of the parasite on the platelets [10]. Oxidative stress damage of thrombocytes has also been implicated in the etiopathogenesis based on the finding of low levels of platelet superoxide-dismutase and glutathione peroxidase activity and high platelet lipid peroxidation levels in malaria patients, when compared to those of healthy subjects [11]. Both nonimmunological destruction [12] as well as immune mechanisms involving specific plateletassociated IgG antibodies that bind directly to the malarial antigen in the platelets have been recently reported to play a role in the lysis of platelets and the development of thrombocytopenia [13]. In clinical trials, recombinant - macro-phage colony stimulating factor (M-CSF) has been known to cause a reversible dose dependent thrombocytopenia. Elevated MCSF levels in malaria, by increasing macrophage activity may mediate platelet destruction in such cases [14]. 


\section{ORIGINAL ARTICLE}

CONCLUSION: In conclusion, absence of thrombocytopenia is uncommon in the laboratory diagnosis of P. Vivax malaria. Thrombocytopenia due to P. vivax malaria is increasing in India but usually disappears with the treatment of disease. Thrombocytopenia in P. vivax has multifactorial etiology, and it has some diagnostic significance. This can be used for predicting presence of malarial infestation even in early stage of the disease. Thrombocytopenia should increase the suspicion of malaria, and multiple peripheral smears or more sensitive tests for detection of parasite should be performed.
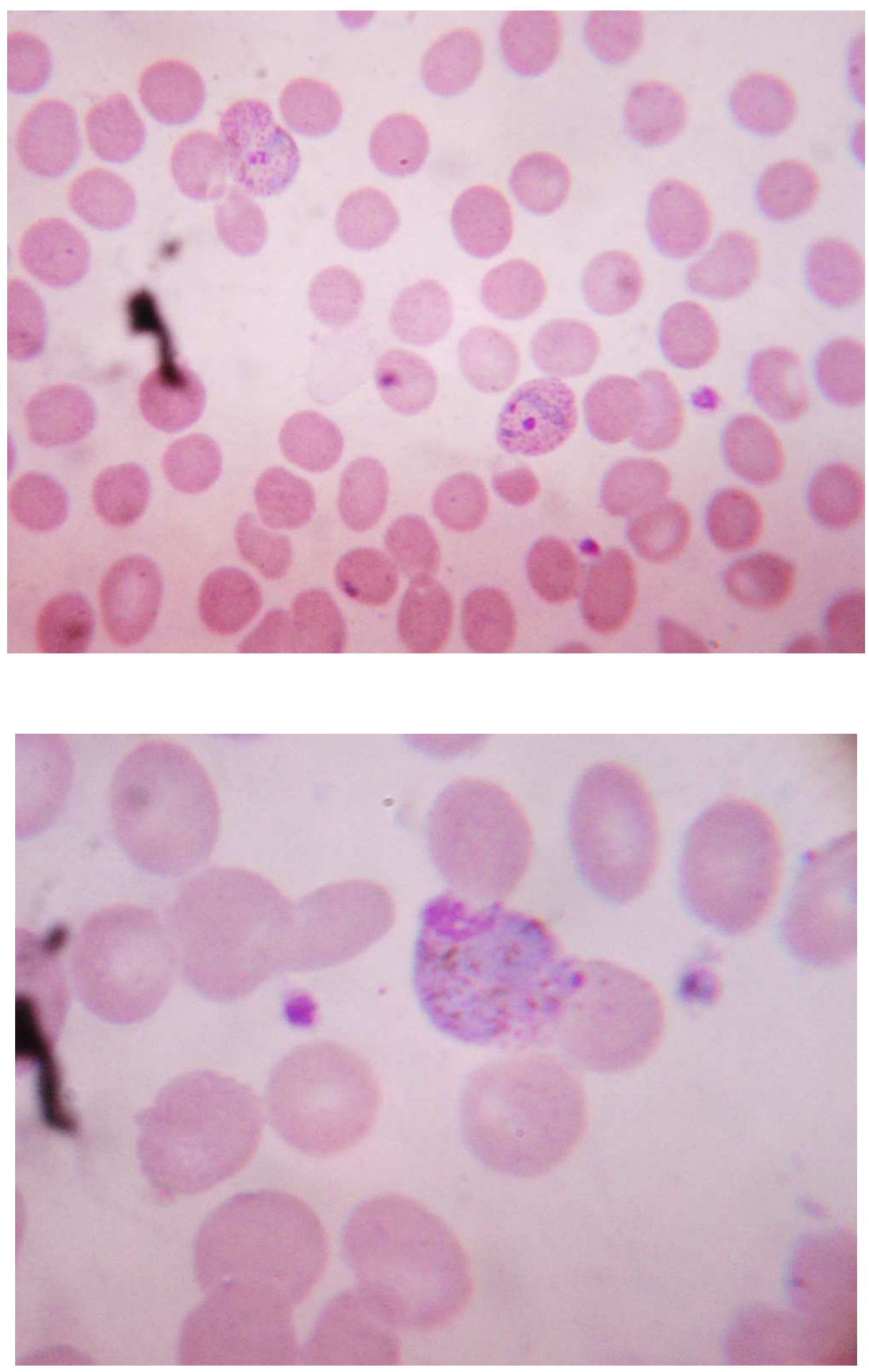


\section{REFERENCES:}

1. Kahl U. Malaria kills over 1 million people every year. Genomic mapping of malaria parasite and mosquito raise hope for a vaccine as well as more effective drugs. Lakartidningen 2003; 100 (12): 1042-47.

2. McNeeley DF, Chu A, Lowe S, et al. Malaria surveillance in New York City, 19911996. nt J Infect Dis. 1998;2:132-6.

3. Khan SJ, Khan FR, Usman M, Zahid S. Malaria can lead to Thrombocytopenia. Rawal Med J 2008; 33: 183-5

4. Looareesuwan S, Davis J.G., Allen D.L., et al.Thrombocytopenia in malaria. Southeast Asian J Trop Med Public Health 1992; 23:44.

5. Martelo O.J., Smoller M., Saladin T.A. Malaria in Americansoldiers. Arch Int Med 1969; 123:383-7.

6. Katira B, Shah I Thrombocytopenia in Plasmodium vivax infected children, J Vect Borne Dis 43, September 2006, pp. 147-9

7. Phulpoto JA, Shaikh AH, Frequency of thrombocytopenia in malarial patients. Medical channel. 2010; 16(4):511

8. Kochar DK, Das A, Kochar S K, Saxena V, Sirohi P, Garg S, Kochar A et al Severe Plasmodium vivax Malaria: A Report on Serial Cases from Bikaner in Northwestern India. Am J Trop Med Hyg February 2009 vol. 80 no. 2 194-8

9. Narang GS, Singla N. Thrombocytopenia and other complications of Plasmodium vivax malaria. Curr Pediatr Res 2011; 15 (2): 117-119

10. Fajardo L.F, Tallent C. Malarial parasites within human platelets. JAMA,1974; 229:12059

11. Erel O, Vural H, Aksoy N, et al. Oxidative stress of platelets and thrombocytopenia in patients with vivax malaria. Clin Biochem 2001; 34 (4): 341-4

12. Looaresuwan S, Davis J.G, Allen D.L, et al. Thrombocytopenia in malaria. Southeast Asian J Trop Med Public Health 1992; 23 (1): 44-50.

13. Yamaguchi $\mathrm{S}$, Kubota $\mathrm{T}$, Yamagishi $\mathrm{T}$, et al. Severe thrombocytopenia suggesting immunological mechanism in two cases of vivax malaria. Am J Hematol 1997; 56 (3):183-6

14. Lee S.H, Looaresuwan S, Chan J, et al. Plasma macro-phage colony stimulating factor and P-selection levels in malaria associated thrombocytopenia. Thromb Haemost 1997; 77 (2): 289-93 